Haben auf Bundes- und Länderebene die zuständigen Politiker, Experten und Fach- und Führungskräfte die Kraft, an der Realisierung des fachlich unbestrittenen Konzepts der ambulanten und stationären Resozialierung (mit gleichwertiger Opferperspektive) festzuhalten, es fortzuentwickeln und die dafür notwendigen rechtlichen, materiellen und politischen Rahmenbedingungen zu gewährleisten?

Oder wird angestrebt bzw. billigend in Kauf genommen, dass Abstand genommen wird vom Konzept der sozialen Integration, dass (vermeintliche) Sicherheit mit der Folge der Ausgrenzung und Stigmatisierung das neue Leitbild wird? Die Bei- spiele in den USA und in Russland zeigen die Folgen: explodierende Gefangenenzahlen, steigende Kosten, kein Rückgang der Kriminalität., Angst vor den im Vollzug nur verwahrten und zur Entlassung anstehenden Gefangenen.

Die Diskussion über die Beibehaltung der Bundeszuständigkeit für die Gesetzgebung für den Strafvollzug oder über ihre Verlagerung auf die Länder wird eine Nagelprobe für diese Richtungsentscheidung werden: bleibt es bei der gesamtgesellschaftlichen Verantwortung und bei einer fachlichen Integration in ein Gesamtkonzept der Strafrechtspflege (mit einer Bundeszuständigkeit für StGb, StPO, JGG, KJHG, StVollzG, U-
HaftVollzG, JugendVollzG etc) oder wird diese Schwerpunktsetzung und fachliche und systematische Integration aufgegeben und 16 verschiedenen Länderregelungen überlassen?

Die Schritte der kriminalpolitischen Feldentwicklung sind noch lange nicht am Ziel angekommen, werden es nie sein, müssen ständig modifiziert und fortentwickelt werden. Rückschläge und Fehlentwicklungen sind nicht zu vermeiden dürfen aber nicht zur Orientierungslosigkeit oder zu populistischen Anpassungen verleiten. Die »Wirkungsorientierte Steuerung « wird mehr und mehr Effizienz und Effektivität ermöglichen.
Wo aber sind die opinion-leader die ihre gesamtgesellschaftliche Verantwortung rational wahrnehmen und Geduld und Stärke mitbringen, diese schwierigen Innovations- und Modernisierungsprozesse abzusichern?

Perspektiven für den deutschen Strafvollzug: mühsame Feldentwicklung in kleinen Schritten oder Ausgrenzungs-Strategie nach amerikanischem Muster?

Bernd Maelicke gehört zu den Heraugebern dieser Zeitschrift und lehrt als Hochschullehrer an der Universität Lüneburg

\title{
Helga Einsele und ihre kriminalpolitischen Wirkungen in der Öffentlichkeit
}

Bernd Maelicke

\begin{abstract}
n NK 2-2005 hat Hannelore Maelicke einen Nachruf auf Helga Einsele geschrieben. Dieser Beitrag wurde im Oktober als Vortrag auf dem 30jährigen Jubiläum des Mutter-KindHeimes der JVA Frankfurt des Vereins für Kinder von Trennungswaisen gehalten.
\end{abstract}

Wenn man die letzten 50 Jahre überblickt, so gehört Helga Einsele zu den wenigen Persönlichkeiten, die in der Öffentlichkeit positiv mit dem Thema Strafvollzug in Verbindung gebracht wurden und dies auch weiterhin werden. Sie hat sich ein Leben lang mit dem Programm eines »Strafvollzugs der positiven $\mathrm{Zu}$ wendung « identifiziert und zu Wort gemeldet - und sie fand in den Medien und bei den Bürgern überwiegend Verständnis und Unterstützung. Was sind die Gründe dafür was können wir von Helga Einsele lernen?

Bei einer solchen Fragestellung muss deutlich getrennt werden zwischen dem Einzigartigen und Persönlichkeitsspezifischen, dass sich mit dem Lebensweg und den Wirkungen von Helga Einsele verbindet und dem Verallgemeinerbaren, Über- tragbaren und Erlernbaren, das als Richtschnur für andere - professionell oder ehrenamtlich Tätige - gelten kann. Als Person ist Helga Einsele ist nur erklärbar durch Verlauf und Historie des 20. Jahrhunderts, das sie nahezu vollständig erlebt und erlitten hat, durch ihr Elternhaus, ihre Erfahrung als Mädchen in Jungenschulen, ihre Begegnung mit Gustav Radbruch, ihre »neuen Erfahrungen « in den USA, durch das Berufsverbot im Dritten Reich, ihre »Lebensentscheidung « für den Frauenvollzug, ihr lebenslanges politisches Engagement, ihre wissenschaftlichen und publizistischen Tätigkeiten. In ihrer vergriffenen Biografie "Mein Leben mit Frauen in Haft « - sie selbst bezeichnet dieses eindrucksvolle Werk als "Niederschrift « (hier ist deutlich die Handschrift ihrer Tochter Nele Löw-Beer zu erkennen) - und in der Festschrift zu ihrem 80. Geburtstag wird deutlich, dass hier eine Frau über viele Jahrzehnte hinweg Handlungsmaximen und humane Maßstäbe gesucht, gelebt und gesetzt hat, die für nahezu alle ihre Mitstreiterinnen und Mitstreiter hohe, teilweise allzu hohe Anforderungen darstellen. Dies wurde auch ein Pro- blem für einige ihrer Nachfolger im Amt der Gefängnisdirektorin.

Am 4. November 1947 begannen für Helga Einsele »fast 28 Jahre einer totalen Inanspruchnahme durch den Beruf mit den oft erschütternden Schicksalen von Frauen « und wie sie es lapidar ausdrückte »durch eine Reihe von meiner Meinung nach dazugehörenden politischen Nebentätigkeiten«. Ähnlich wie Radbruch nannte sie dieses Leben ein »entpersönlichendes Klosterleben « - aber, wie sie anfügt »vor allem geformt durch die Beziehung zu einer im hohen Maß verpflichtenden und in enger Verbindung mit nahe stehenden Mitarbeiterinnen zu erfüllenden Aufgabe«.

Übrig blieb damals (im Jahr 1947) »das Heimweh nach dem Gefährten in Österreich und eine vom schlechten Gewissen überschattete Verbundenheit mit einem kleinen Mädchen, das mehr und mehr zur Partnerin wurde « - und dies bis zu ihrem Tod im begnadeten Alter von 94 Jahren. Wie bereits erwähnt, ist diese partnerschaftliche Mitwirkung unter anderem deutlich nachzuverfolgen in den zahlreichen Veröffentlichun- gen von Helga Einsele, an denen Frau Nele Löw-Beer hilfreich unterstützend mitgewirkt hat.

Ihr »Leben um der Überzeugung willen «, ihr »Schwimmen gegen den Strom «, ihre reformerischen Aktivitäten in der Vollzugsanstalt für Frauen - all dies ist immer wieder beschrieben und gewürdigt worden und soll deshalb an dieser Stelle nicht wiederholt werden. Ihre Wirkungen halten auch 30 Jahren nach dem Ausscheiden aus dem aktiven Dienst immer noch an - ihre konzeptionellen und vollzugspolitischen Vorstellungen und Forderungen sind noch lange nicht erfüllt! Und erst recht nicht ihr Ruf und der ihres Lehrers Radbruch nach etwas Besserem als dem Strafrecht oder nach einer strukturellen Reduzierung der Zahl der U- und Strafgefangenen bei gleichzeitigem Ausbau von ambulanten Behandlungs- und Wiedereingliederungsangeboten.

Viele ihrer Ideen und Projekte sind sogar gefährdet - so sind z.B. die Zahlen des offenen Vollzuges und der vorzeitigen Entlassung rückläufig, Personal wird abgebaut, für ambulante Dienste und Nachsorgeein- 
richtungen - insbesondere für die Anlaufstelle für straffällig gewordene Frauen - werden die Mittel gekürzt oder gestrichen. Helga Einsele hat in ihrem letzten Grußwort an die Vollzugsbranche im Jahr 2004 diesen Tatbestand wie folgt charakterisiert: »Der Stern von Freiheit und Humanität leuchtet offensichtlich an wechselnden Orten und mal heller und mal schwächer«.

\section{Strafvollzug in den Medien - eine eigene Welt}

Warum gibt es immer und immer mehr in den letzten Jahren diese Hinwendungen von Kriminalpolitikern und Medien zu populistischen Aussagen und Gesetzesinitiativen, die weder kriminologisch noch vollzugswissenschaftlich betrachtet als »rational « bezeichnet werden können? Die bewusst und vorsätzlich an das »Irrationale « im Umgang mit der Kriminalität, mit dem Thema der inneren Sicherheit appelieren?

Holle Löhr, Leitende Oberstaatsanwältin in Itzehoe, hat in einem wichtigen Beitrag das Spannungsfeld zwischen dem Thema der Resozialisierung und seiner öffentlichen Darstellung analysiert und beschrieben (Handbuch der Resozialisierung, S. $529 \mathrm{ff}$.).

Sie stellt fest, dass die Themen Kriminalität und Strafvollzug in den Medien nach drei Gestaltungsprinzipien dargestellt werden:

\section{- Selektion}

\section{- Vereinfachung}

\section{- Sensationsinteresse.}

Während z.B. die Gewaltdelikte zusammen $5,3 \%$ in der PKS ausmachten (1985) beschäftigten sich im gleichen Jahr 66,3 \% der Beiträge von ausgewählten Zeitungen mit dieser Thematik. Mord und Totschlag $(0,1 \%$ laut PKS) nahmen $21,7 \%$ Umfang in der Presseberichterstattung ein.

Über den Strafvollzug wird fast nur in Krimis oder Serien berichtet, die wiederum nur als Zerrbild bezeichnet werden können. Die RTLSerie über den Frauenvollzug stellt das Personal inklusive Anstaltsleiter überwiegend als Erpresser und Vergewaltiger dar. Sozialarbeiter, Psychologen, der gesamte Behandlungsvollzug kommen weder thematisch noch personell vor.

Diese mediale Darstellung ist orientiert an den Gesetzen der Marktwirtschaft und der Konkurrenz, entscheidend sind Verkaufszahlen und Einschaltquoten.

Bei den Zuschauern rufen diese Darstellungen Empörung und Abscheu vor den Tätern sowie das Bedürfnis nach Bestrafung und Ausgrenzung, nach Wegsperren hervor. Differenzierende und rationale Beiträge finden sich nur in wenigen anspruchsvollen Medien oder in Rundfunk- oder Fernsehsendungen zu ungünstigen Sendezeiten.

Für 95 \% aller Bürger sind die Massenmedien die einzige Informationsquelle über Kriminalität. So wird eine Kunstwelt geschaffen, die wenig oder nichts mit der Wirklichkeit zu tun hat.

\section{Die öffentliche Meinung zu Strafe und Strafvollzug}

Es ist eine logische Folge, dass die öffentliche Meinung sich in einer großen Diskrepanz zu den gesetzlichen Grundlagen von Strafe und Strafvollzug und $\mathrm{zu}$ wissenschaftlichen Erkenntnissen befindet.

Das Gesetzt versteht Strafe als gerechten Schuldausgleich unter Berücksichtigung der Schwere der Tat, der Folgen für das Opfer und der individuellen Schuld des Täters. Die öffentliche Meinung dagegen äußert sich fast immer aus der Sicht der potenziellen Opfer. Sie ruft nach harten Strafen, ist für lebenslanges Wegsperren, ist gegen Lockerungen und vorzeitige Entlassung. An die Abschreckung wird fest geglaubt, das Konzept der Resozialisierung findet immer weniger Unterstützung.

\section{Der publizistisch-politische Verstärkerkreislauf}

Holle Löhr stellt fest, dass es zwischen den Medien und den verantwortlichen Politikern zu Wechselwirkungen kommt, die diesen Prozess der verzerrten Realitätswahr- nehmung und der irrationalen Problembearbeitung im Sinne einer wachsenden Dramatisierung noch weiter verstärken.

Die selektive, vereinfachende und sensationsorientierte Berichterstattung führt zu steigender Kriminalitätsfurcht - über diese wird wiederum in den Medien mit dem Vorwurf an die Politik berichtet, dass diese ihre Aufgabe nicht wahrnehme und dass deshalb ein Politikwechsel (= Politikerwechsel) erforderlich sei.

Politiker wollen als Problemlöser, als Gestalter, als Macher wahrgenommen werden, die nicht dem Rat von Experten und Wissenschaftlern folgen, die viel zu viel problematisieren und differenzieren.

So kommt es immer wieder $\mathrm{zu}$ neuen Gesetzesinitiativen, die ein schnelleres und härteres Vorgehen gegen die Kriminalität und gegen Kriminelle versprechen. Effizienzforschungen, ob die versprochenen Wirkungen auch eintreten, werden nicht veranlasst. Ebenso keine Kosten-Nutzen-Vergleiche z.B. über Effektivität und Effizienz von freiheitsentziehenden Maßnahmen im Vergleich zu ambulanten.

\section{Das veränderte kriminal- politische Kraftfeld}

Das folgende Schaubild hat sich gut bewährt, um die Kräfte in ihrer Wechselwirkung zu analysieren, die im Wesentlichen die öffentliche Diskussion und die praktische Entwick-

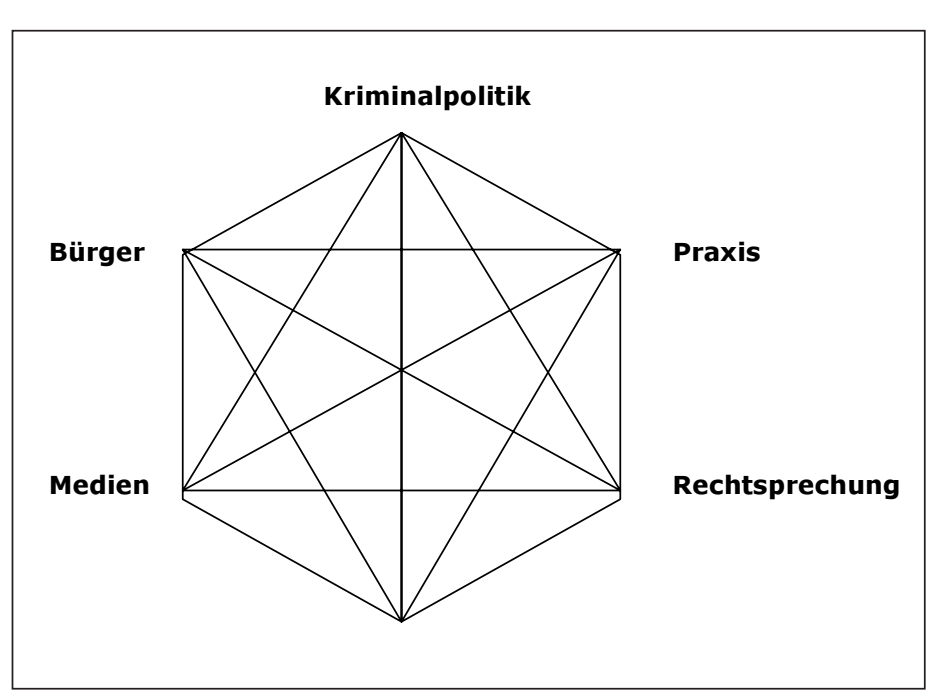

lung in den verschiedenen Arbeitsfeldern der Kriminalpolitik wesentlich beeinflussen.

Im Vergleich zu den 70er- und 80er-Jahren haben sich gravierende Änderungen im kriminalpolitischen Kraftfeld ergeben:

Zurzeit der Strafvollzugsreform gab es eine hohe Übereinstimmung zwischen der Praxis, der Rechtsprechung, der Wissenschaft, den Medien, den Bürgern und der Kriminalpolitik. Das Konzept der »Sozialen Strafrechtspflege entsprach dem Grundgesetz und dem öffentlich vorherrschenden Menschenbild in den Medien, Kirchen und Verbänden.

Heute wird über »Monster «, »Irre « und »Kannibalen « berichtet, die für immer wegzusperren sind, keine Freistunden, kein schmackhaftes Essen, keine wohnliche Zellenausstattung und keine Fernsehunterhaltung haben sollen.

Die entscheidende Wechselwirkung findet heute zwischen den Medien und den Kriminalpolitikern statt. Die übrigen Stimmen im Kraftfeld sind kaum noch zu vernehmen. Deutlich wird dies z.B. beim Thema Bundeszuständigkeit für die Vollzugsgesetzgebung.

Helga Einsele und das kriminalpolitische Kraftfeld

In den Jahren, in denen Helga Einsele ihre größte Wirksamkeit erreichte, 
verkörperte sie selbst so etwas wie ein kriminalpolitisches Kraftfeld.

Sie war eine hoch geachtete Anstaltsleiterin, die über Jahrzehnte hinweg dauerhaft und unermüdlich ihre Anstalt zu einer viel beachteten und beispielhaften Reformeinrichtung fortentwickelt hat.

Sie hatte als Mitglied der Strafvollzugskommission das kommende Bundes-Strafvollzugsgesetz ganz wesentlich mitgeprägt - nicht nur in Bezug auf die Regelungen über den Frauenvollzug und die gemeinsame Unterbringung von Müttern und ihren Kindern, sondern als erfahrene Praktikerin bezogen auf das gesamte Regelwerk.

Als Gutachterin für das Bundesverfassungsgericht und den Europarat nahm sie Einfluss auf die Rechtsprechung und die Entwicklung internationaler Standards.

Sie publizierte in Fachzeitschriften und wissenschaftlichen Journalen, übernahm wissenschaftliche Begleitungen und wurde zur Honorarprofessorin an die Universität Frankfurt a.M. berufen

Die Journalisten liebten und achteten sie: Sie formulierte brillant und glasklar, unmissverständlich trat sie für ihre Sache ein. Thema und Person passten zusammen - es gab keine Trennung zwischen beruflichem Engagement und privatem Leben. Der vollzugsspezifische Zynismus, der vielfach für professionelles Leben in dieser Institution voller Widersprüche unverzichtbar erscheint, war ihr Gegner, den sie bei jeder Gelegenheit bekämpfte - oder besser, der in ihrer Gegenwart nicht geäußert werden durfte. Also eine öffentliche Persönlichkeit, die ihresgleichen sucht - zumal in heutigen Zeiten. Sie wurde dauerhaft begleitet von Journalisten wie Ulrike Holler vom Hessischen Rundfunk und entfaltete so zusammen mit dieser eine große Wirkung in Hinblick auf das Verständlichmachen und das Werben für eine rationale Kriminalpolitik.

Sie trat jahrzehntelang in ungezählten öffentlichen Veranstaltungen, in Volkshochschulen und Arbeitskreisen auf, hatte nicht nur über ehrenamtliche Helfer engen Kontakt zu den Bürgern, sondern arbeitete auch immer wieder eng mit Bürgerinitiativen zusammen. So war sie verbunden mit der Humanistischen Union und vor allem mit dem Kinderheim Preungesheim e.V. und engagierten Frauen wie Jutta Frost, Helga Matthiesen und Dorothee Vorbeck, um nur einige wenige Namen zu nennen.

Und sie hatte Kontakt zu Kriminalpolitikern auf Landes- und Bundesebene, trat in Hearings im Landtag und im Bundestag auf. Dabei immer aufrecht und unbestechlich, untaktisch, manchmal auch zornig und verletzbar, immer konkret, nie abstrakt.

Sie kannte jedoch auch den Preis, den sie und ihr Umfeld bezahlen mussten. Sie erlebte erbitterte Gegnerschaft, war und fühlte sich allein gelassen in kritischen Situationen.

Aber sie realisierte immer und gegenüber jedermann »positive $\mathrm{Zu}$ wendung «. Jeder bekam immer wieder eine neue Chance, niemand wurde auf Dauer abgeschrieben.

Schwimmen gegen den Strom - um der Überzeugung willen

Was bleibt?

Jeder muss seinen eigenen Weg gehen - bestimmt durch Herkunft, gesellschaftliche Rahmenbedingungen, Ausbildung, Freundeskreis, Partner, wichtige Ereignisse, glückliche und weniger glückliche Umstände.

Willi Brandt wusste um die begrenzten Möglichkeiten, die eigene Wirksamkeit zu bestimmen und gezielt einzusetzen. Nur wenn das richtige Thema, die richtige Person und der richtige Zeitpunkt zusammenkommen, kann man optimale Wirkung erzielen.

Helga Einsele hat ein Leben - ihr Leben - investiert und durchgehalten, um für ihr Thema der Resozialisierung durch positive Zuwendung zu kämpfen. Dabei gab es glückliche Situationen und Gelegenheiten, in denen Thema, Person und Zeitpunkt optimal zusammenpassten. Aber es gab auch viele Situationen, in denen sie allein gelassen war mit ihrem
Thema, in denen »die Sonne der Resozialisierung « vom Untergang bedroht war.

In ihrem Resümee in ihrer Niederschrift bleiben ihre Zweifel dokumentiert:

Es hat sich für sie erwiesen, dass es im Strafvollzug immer wieder - über das Notwendige hinaus - zu Regressionen und Repressionen kommen wird und die Gefahr erhalten bleibt, dass auch diese Form von Machtausübung über Menschen immer mehr Schaden als Nutzen verursachen wird, dass es also notwendig ist, auf Machtausübung sowohl politisch wie rechtlich soweit wie irgend möglich im Zusammenleben von Menschen zu verzichten.

Und sie schließt: »Trotz aller berechtigten Zweifel an wirklichen Erfolgen möchte ich zum Schluss noch sagen, ich bereue dieses Leben nicht«.

Diese Botschaft können wir nachvollziehen: Es bleiben berechtigte Zweifel an wirklichen Erfolgen selbst wenn man/frau wie Helga Einsele ein Leben investiert. Wichtig ist das »strebende Bemühen « - die Bereitschaft zum Schwimmen gegen den Strom - um der Überzeugung willen in enger Verbindung zu den Mitstreiterinnen und Mitstreitern und mit einem Menschenbild de positiven Zuwendung.
Ohne die Unterstützer kann man sich auf Dauer nicht gegen den Mainstream durchsetzen: Was wäre das Mutter-Kind-Heim ohne den Kinderheim Preungesheim e.V., ohne begleitende Supervision, ohne wissenschaftliche Begleitung, ohne die Förderung der Fortbildung der Mitarbeiter, ohne Finanzierung von Sachmitteln und ohne finanzielle Unterstützung von Müttern und Kindern weit über die Entlassung hinaus?

In einer Zeit des rapiden Wandels und der zunehmenden Verunsicherung ist Wirksamkeit auf Dauer ohne ein flexibles Netzwerk, ohne ein enges Zusammenwirken über die Grenzen von staatlichen, freien und ehrenamtlichen Initiativen hinaus nicht möglich. Gerade in Krisenzeiten wird gelebte Solidarität unverzichtbar. Deshalb auch im Namen von Helga Einsele an dieser Stelle und zu diesem Zeitpunkt Dank und Anerkennung für die Mitwirkung und Unterstützung an den Kinderheim Preungesheim e.V. und an al die Personen und Institutionen, die nunmehr seit über 50 Jahren dafür sorgen, dass nicht nur im Frauenvollzug und in der ambulanten Nachsorge die "Sonne der Resozialisierung « weiterhin leuchtet und nicht vom Untergang bedroht ist.

Bernd Maelicke lehrt an der Fachhochschule Lüneburg und ist Mitherausgeber dieser Zeitschrift

\section{»Apokryphe« Disziplinar- maßnahmen im Strafvollzug}

Joachim Walter

\section{Rechtstatsachen}

M ie jeder Vollzugspraktiker und erst recht jeder Gefangene weiß, werden im deutschen Strafvollzug Disziplinarmaßnahmen, früher Hausstrafen genannt, sehr häufig verhängt. Diese repressiven Sanktionen sollen dazu dienen, die Ordnung in den Justizvollzugsanstalten aufrecht $\mathrm{zu}$ erhalten. Insbesondere die Untersuchungen von
Dünkel (1996, 101 ff) der auch Länder- und Anstaltsvergleiche angestellt hat, haben gezeigt, dass die pro 100 Gefangene der Jahresdurchnittsbelegung verhängten Disziplinarmaßnahmen enorm schwanken. Im Bundesdurchschnitt handelte es sich im Jahre 1994 um 54,4 Maßnahmen pro 100 Gefangene - gewiss hoch genug. Andererseits hat diese Rate in diesem Jahr beispielsweise in Brandenburg 13,2 Maßnahmen, im Saar- 